\title{
túnel bajo el agua
}

E. STAMHUIS, ingeniero

\section{silm opesis}

$575 \cdot 5$

El ingeniero A. Eggink describió, en un trabajo publicado últimamente, un nuevo tipo de túnel bajo el agua para canalizar la circulación rodada. En este trabajo se desarrolla un analisis sobre las consideraciones que han jugado un papel predominante en la redacción del proyecto de ejecución de un túnel subacuo. Acompañan al trabajo los resultados referentes a los distintos cálculos realizzdos por el Instituto Central de Inves. tigaciones Científicas de Holanda,

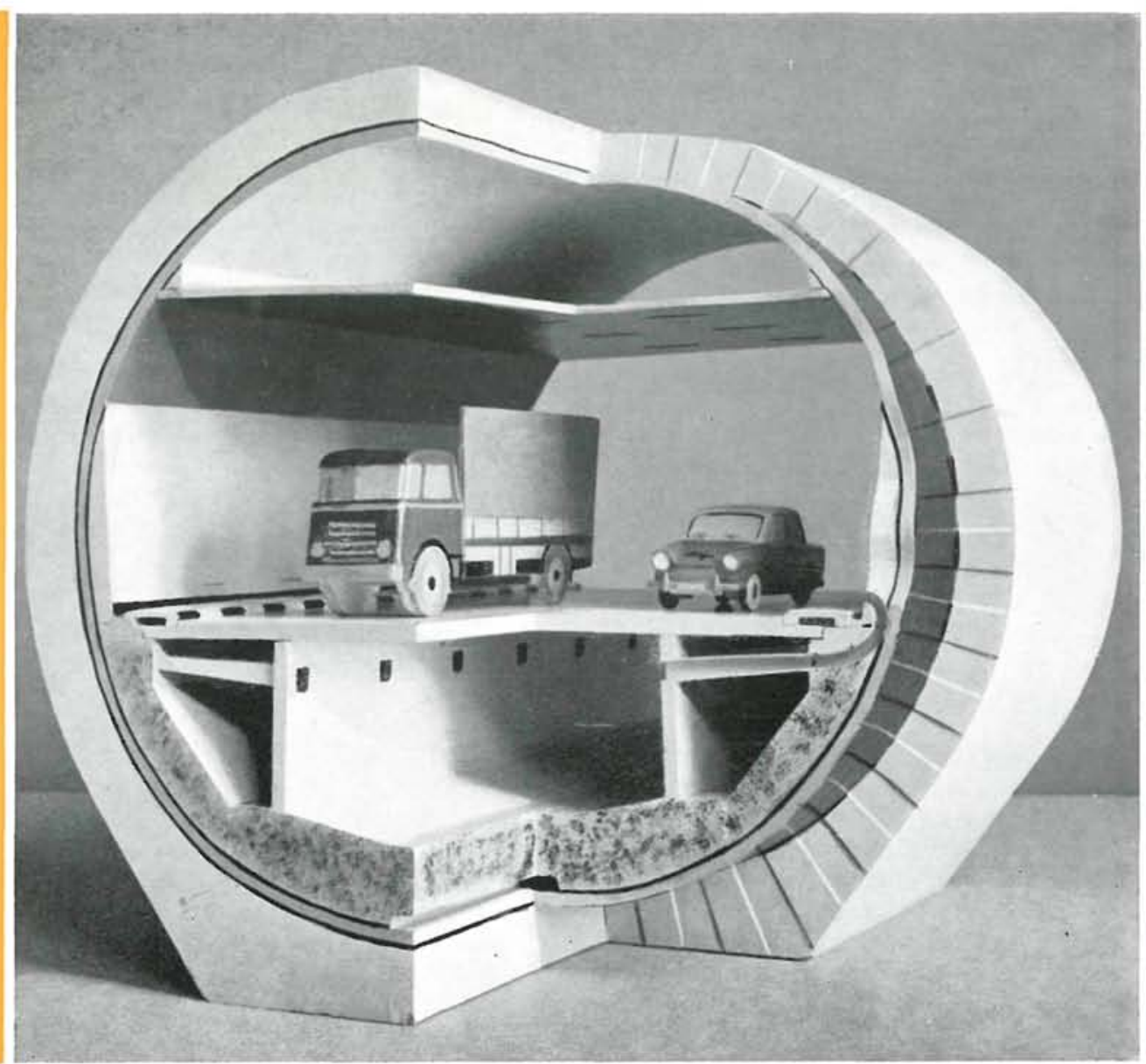

Fig. 1.-Maqueta de un trozo de túnel.

En este trabajo se analizan los resultados obtenidos de un estudio teórico llevado a cabo por el Instituto Central de Investigaciones Científicas de Holanda sobre túneles sumergidos en el agua, con objeto de esta. blecer normas generales para proyectar este tipo de túneles con ventajas sobre los actualmente existentes. En primer lugar se estudian la
ción del proyecto de ejecución.

Seguidamente se analiza la forma, secciones apropiadas y métodos constructivos, comparándolos con los utilizados en la construcción de otros túneles de esta naturaleza en América. Son objeto de particular atención los procedimientos en los que la línea general constructiva se basa en la prefabricación y montaje de elementos estructurales preparados en taller, su transporte y colocación en obra.

Como detalles complementarios el autor se extiende en consideraciones del juego de presiones del suelo y agua, empujes de flotación y los casos en que aparecen flexiones y esfuerzos cortantes en los distintos tramos en que se puede subdividir la longitud total del túnel.

Se esfuerza el autor en dar una carácter práctico a las aplicaciones que del estudio teórico se pueden derivar para la realización de la obra.

También se describen: unos pasadores especiales que sirven para solidarizar los dos tubos de que se compone el túnel; las juntas entre dos trozos distintos del mismo, su impermeabilización y la flexibilidad que con llos se logra.

Finalmente, el autor resume su exposición teórica con una serie de observaciones y consejos aplicables a estas construcciones. 
La elección de la forma del perfil transversal depende de diferentes factores, entre los que podemos citar como más importantes: el espacio interior, la resistencia, la impermeabilización y ventilación, y los métodos constructivos.

\section{Espacio interion}

Para evitar los efectos del empuje de flotación, el peso del túnel sumergido debe equilibrar al referido empuje. Si llamamos $A$ al espacio interior necesario, y $\gamma$ representa la densidad, la cantidad de hormigón precisa vendrá expresada en la forma siguiente:

$$
\frac{A \cdot \gamma \text { densidad del agua }}{\gamma \text { del hormigón }-\gamma \text { del agua }}=0,7 \mathrm{~A} \text { aproximadamente. }
$$

La cantidad total de hormigón ha de repartirse lo más eficientemente posible a lo largo del perfil longitudinal del terreno de sustentación.

\section{Resistancia}

Las cargas más importantes son: el peso propio de la estructura, la presión del agua y la presión efectiva en el suelo circundante. La resultante total del peso propio y la presión del agua es aproximadamente nula. La distribución de estas cargas no debe depender de las deformaciones del cuerpo estructural del túnel. La distribución de la presión efectiva en el suelo depende, sin embargo, de estas deformaciones.

El empleo de secciones transversales más o menos rectangulares da origen a presiones en la estructura como consecuencia del peso propio y la presión del agua que se presentan aumentadas al descender bajo el agua. Las presiones en el túnel debidas a las presiones en el terreno aumentan al aumentar la profundidad del suelo de apoyo y, por tanto, el porcentaje de refuerzo oscila alrededor de 1,5 por 100, limitando, por consiguiente, la profundidad de ubicación, si es que desea hallarse, dentro de la posibilidad de la construcción del túnel, de acuerdo con el método denominado tubo sumergido o también de trinchera abierta.

De emplear secciones transversales circulares con espesor uniforme de muros, la carga por peso propio y la presión del agua originan, únicamente, tensiones de compresión pura en los muros del túnel. Esto puede comprobarse sencillamente.

La presión en el suelo da lugar a la presencia de momentos flectores y esfuerzos cortantes. Estas tensiones resultan pequeñas debido a que las presiones en el suelo se ajustan entre sí en forma favorable a las deformaciones por ellas causadas en el cuerpo estructural del túnel. Como resultado de todo ello, el estado tensional no es solamente favorable, sino casi indiferente para la ubicación del túnel, siempre que se refiera a la profundidad del agua y la carga sobre el terreno de sustentación del túnel. El porcentaje de refuerzos resulta, por ello, bajo, es decir, del orden de 0,5 por 100 . Todo esto se traduce en economías de consideración, aun tratándose de profundidades adicionales de unos 60 metros.

\section{Impermentbilizcación y ventilación}

Aunque la forma circular del túnel presenta una sección transversal de distinta resistencia, los otros factores anteriormente mencionados que determinan la elección de la sección transversal-la impermeabilización, la elección del sistema de ventilación y el método constructivo-influencian igualmente el coste.

El caso del túnel Baytown, de Texas, nos muestra un ejemplo. Aquí se tenía la posibilidad de poder elegir entre dos tipos de construcción: Uno de ellos de acuerdo con los procedimientos americanos de doble virola de acero ccmo se observa en la figura 2, y el segundo, constituyendo una alternativa de la solución con un simple tubo en cuyo interior van los muros (ver fig. 3). Este último tipo se estimó que era un 15 por 100 más bajo en precio que el correspondiente a la solución clásica.

Aunque las relaciones del precio del acero y el hormigón difieren entre Holanda y los Estados Unidos, puede asegurarse que una limitación en el consumo de acero constituye siempre un ahorro en su aspecto económico.

La elección del sistema de ventilación también afecta a la sección transversal. Por consiguiente, el espacio interior circular del túnel de Baytown ha resultado muy eficiente para el empleo de un sistema de ventilación en el que el aire fresco es inyectado en el interior por medio de una simple estación de 


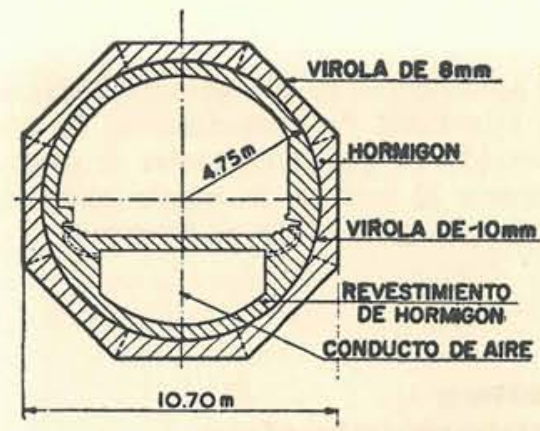

Fig. 2.-Sección de túnel típico americano.

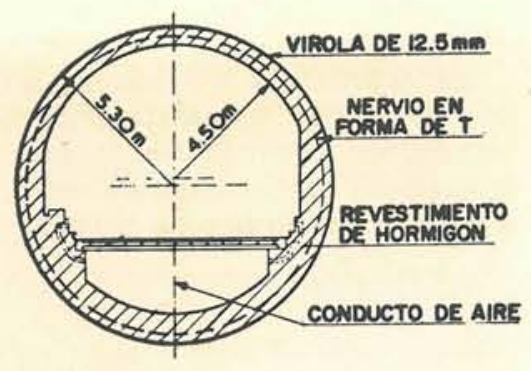

Fig. 3.-Túnel de Baytown.

ventilación para toda su longitud de $918 \mathrm{~m}$, utilizando como canalización del aire viciado el espacio arqueado reservado al tráfico. En oposición con el coste suplementario que motiva una sección transversal más espaciosa, se tiene la ventaja de la eliminación total de una estación de ventilación y, con ello, un simple sistema operacional y la posibilidad ulterior de una modiflcación del sistema de ventilación si las necesidades así lo exigiesen.

\section{Comsst?mencelón}

Se comprenderá que es deseable orientarse hacia una sección transversal circular y, consecuentemente, hacia una economía, limitando a un mínimo el consumo de acero, pero, en este caso, el método constructivo juega un papel importante.

Con la construcción de un túnel de forma circular, la camisa metálica de impermeabilización, prefabricada, se utiliza como un encofrado para el hormigonado del cuerpo estructural del túnel. De esta forma la camisa metálica no solamente tiene una función de impermeabilización, sino también la de portadora de una carga, motivo por el cual se la refuerza con anillos en forma de nervios. El empleo del asfalto como material de impermeabilización no es posible, puesto que este material no tiene propiedades resistentes. Las fases de la construcción son, por tanto, opuestas a las del empleo del acero, ya que el asfalto se aplica recubriendo el cuerpo prefabricado del túnel, constituyendo así un buen procedimiento para obtener la impermeabilización. Si se trata de una sección transversal circular, este último método es de difícil aplicación y caro; hasta la actualidad, y dentro de nuestros conocimientos, ha sido aplicado únicamente en el túnel Oakland Alameda, construido en 1926-1928, sin que haya sido aplicado después en ninguna parte.

En un proyecto preliminar para un túnel bajo el río Riachuelo, de Buenos Aires, y para otro túnel bajo el río Guaiba, de Puerto Alegre (Brasil), se estudió un método para sustituir la camisa metálica por un tubo de $10,40 \mathrm{~m}$ de diámetro, de hormigón pretensado, con un muro de $15 \mathrm{~cm}$ de espesor, prefabricado en trozos de $4,60 \mathrm{~m}$ de longitud.

Un proyecto redactado por dos gabinetes técnicos consultivos, uno de ellos americano y el otro belga, no ha podido realizarse por no llegar a su debido tiempo a una solución satisfactoria para la unión de los elementos de hormigón del tubo y los muros del mismo que debían hormigonarse en el interior del túnel.

La prefabricación de elementos con su montaje sobre un dique seco ha sido aplicada a gran escala en la construcción del túnel del Hampton Roads, de Virgina (U. S. A.), cuya sección es muy similar a la propuesta en este estudio y la prefabricación fue empleada, en su mayor parte, para la preparación de las virolas metálicas del túnel.

No resulta evidente, por sí mismo, que la aplicación de los métodos que utiliza la prefabricación resulten siempre con ventajas económicas sobre los procedimientos tradicionales.

Esto se puede comprobar en el caso de construcciones en general, así como en las de grandes luces y, todavía con mayor facilidad, en las aplicaciones de la ingeniería hidráulica donde las estructuras son, generalmente, de tipo pesado. Sin embargo, el proyecto de una estructura hidráulica conserva su propio carácter, que es muy diferente de aquel que distingue a la ediflcación industrial.

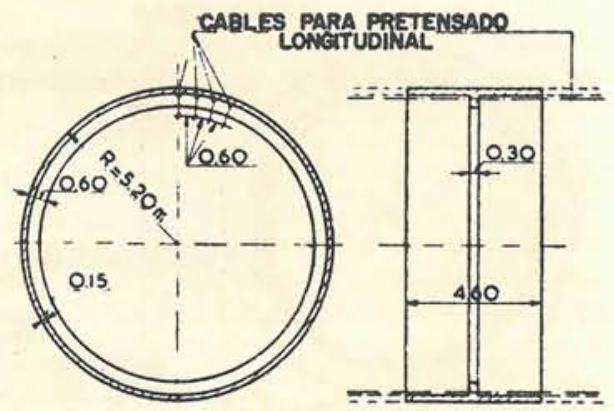

Fig. 4.-Túnel pretensado. 
La experiencia nos enseña, no obstante, que aun tratándose de aplicaciones de la ingeniería hidráulica, los costes de construcción pueden ser reducidos, llegando a soluciones de normalización de determinados elementos de la estructura. Una de las condiciones consiste en que estas partes se prefabriquen en cantidades suflcientes, condición ésta que puede extenderse al caso de la construcción de un túnel, puesto que su estructura presenta una sección uniforme a lo largo de toda su longitud.

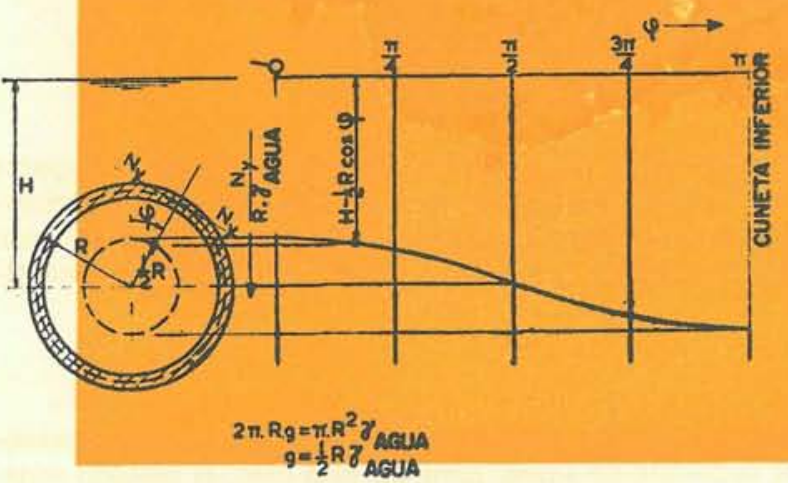

Fig. 5.-Paso propio del tubo.

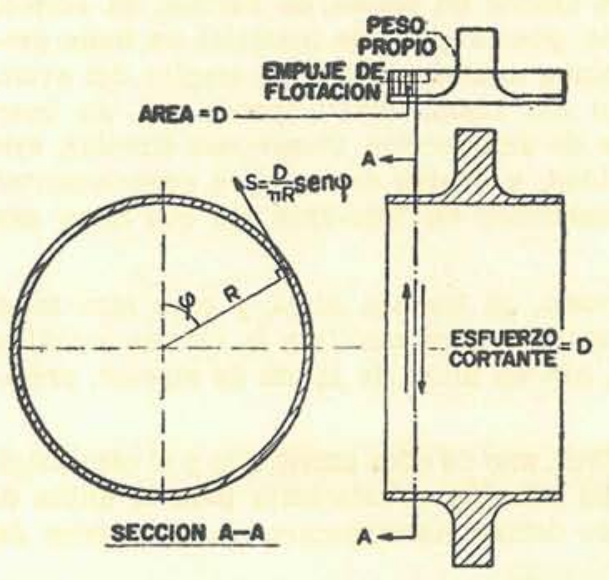

Fig. 6.-Sección con viga anular o nervio.

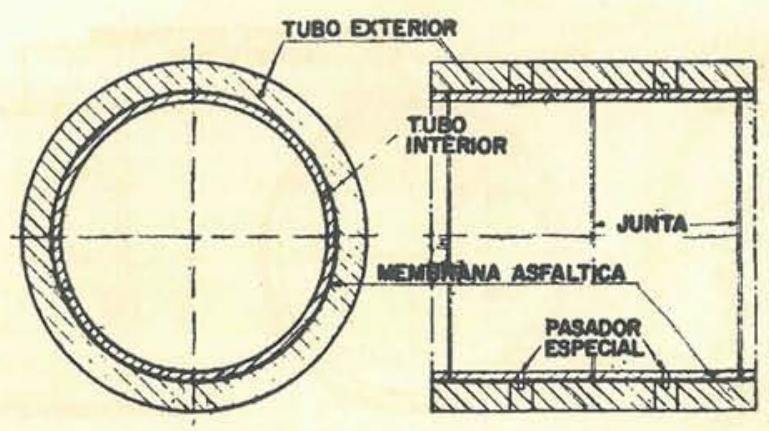

Fig. 7.-Tubos concéntricos.

\section{Deteralless

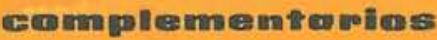

Aparte de la influencia de la presión ejercida por el terreno, la sección mostrada en la adjunta figura 2 se encuentra libre de flexiones si su propio peso iguala al empuje de flotación motivado por el agua. Este fenómeno es extensible a cada una de las unidades lineales del cuerpo estructural del túnel o tubo. También es posible, sin embargo, subdividir el material longitudinalmente en la dirección del túnel de diferentes formas, con lo que se pueden conseguir trozos que siguen conservando secciones transversales libres de efectos de flexión. Las condiciones únicas son: que sobre cierto trozo de túnel, que ha de ser prefabricado, la carga total por peso propio sea igual al empuje total de flotación provocado por el agua, y que cada uno de estos trozos ha de ser considerado como un cuerpo en rotación. En lugar de tensiones de compresión en los anillos, se presentan tensiones similares a las de una membrana y, por tanto, la diferencia entre el peso propio y el empuje de flotación del agua en la dirección del túnel resulta ser igual al esfuerzo cortante transferido de acuerdo con una curva senoidal de una a otra sección.

En la figura 6 la sección transversal tipo es suficientemente fuerte para resistir la presión provocada por el agua, mientras que su peso se halla concentrado en una viga de forma anular.

En la figura 7, la referida viga sirve para formar un segundo tubo alrededor del primero. Entre estos tubos queda espacio sufciente para la impermeabilización, constituida por una membrana asfáltica. El tubo exterior protege a esta capa esfáltica, a la vez que contribuye a aumentar el peso propio. Los dos tubos se hallan en el caso de tensiones de membrana. El tubo exterior es capaz, por si mismo, de absorber las presiones creadas por el terreno circundante.

Debido a las grandes diferencias de rigidez respecto a las presiones radiales en la proximidad de la unión interior, entre los dos tubos tiene lugar un desarrollo más bien 


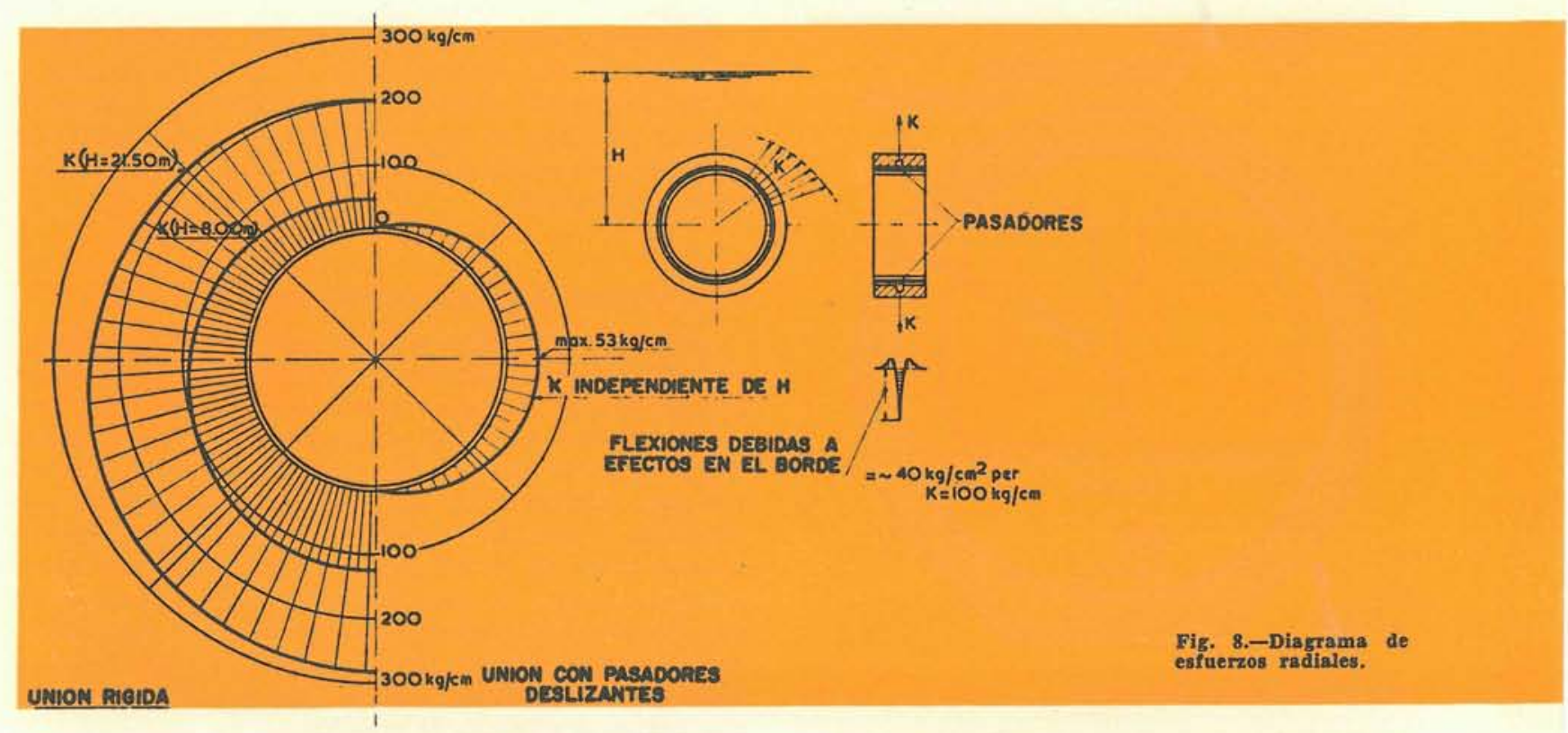

significativo y disturbante de momentos que se hacen sentir en la dirección axial del túnel. Estos efectos se eliminan, en la medida de lo posible, logrando una transferencia de esfuerzos por un sistema de pasadores especiales proyectados para permitir un movimiento radial entre las paredes de los tubos. Para poderse realizar esta transferencia de esfuerzos es necesario y suflciente la transmisión de esfuerzos cortantes que siguen una ley similar a la de una curva senoidal y de dirección tangencial.

Con objeto de lograr trozos transportables, el túnel se subdivide en varios trozos. Para el refuerzo del tubo interior durante su transporte y montaje, una parte del tubo exterior se utiliza como una viga anular para los trozos del tubo interior. Los cálculos del estado tensional de membrana motivado por la carga propia y la presión del agua se efectúan con mayor sencillez siguiendo los métodos básicos de la teoría de vigas, pues se puede considerar al tubo inferior como si se tratase de una viga de corta longitud y grandes alas, mientras que al tubo exterior como si se tratase también de una viga de pequeña longitud libremente soportada en sus dos extremidades. A los esfuerzos así determinados han de añadirse los de tipo anular originados por las componentes de las cargas radiales.

Las reacciones de los tubos, tratándose de estas vigas de tipo anular, subdivididas de acuerdo con la ley de una curva senoidal, son iguales y de direcciones opuestas, es decir, los dos tubos se soportan mutuamente.

\section{Aprifcectomes prodicticens}

Una de las posibles aplicaciones con carácter práctico de estas construcciones, en las que son aplicables las consideraciones teóricas, se indica en las flguras 1 y 9 , que se refleren a un túnel para paso de vehículos y otro para el paso de un ferrocarril.

Para estos proyectos se erige un espesor tal para el tubo exterior, que el peso total, incluyendo las partes interiores de la estructura y el balasto, sea un poco mayor que el empuje de flotación provocado por el agua.

Los elementos del tubo interior tienen una longitud de $5 \mathrm{~m}$ y se hormigonan colocando los encofrados verticalmente. Después de desencofrar y endurecer el hormigón se aplica una doble capa de asfalto reforzada con otras de fleltro o flbras. Después de todo esto, los elementos así preparados se agrupan entre si convenientemente por medio de pasadores especiales para formar un anillo prefabricado. Los elementos así obtenidos experimentan después un giro de $90^{\circ}$ y son transportados al dique seco o a las plataformas flotantes, donde estos anillos se colocan uno al lado de otro y se acoplan convenientemente, utilizando guarnición de goma para las juntas rellenadas a presión. Finalmente, se colocan las armaduras del tubo exterior y se procede a hormigonar la parte que corresponde a este último. 


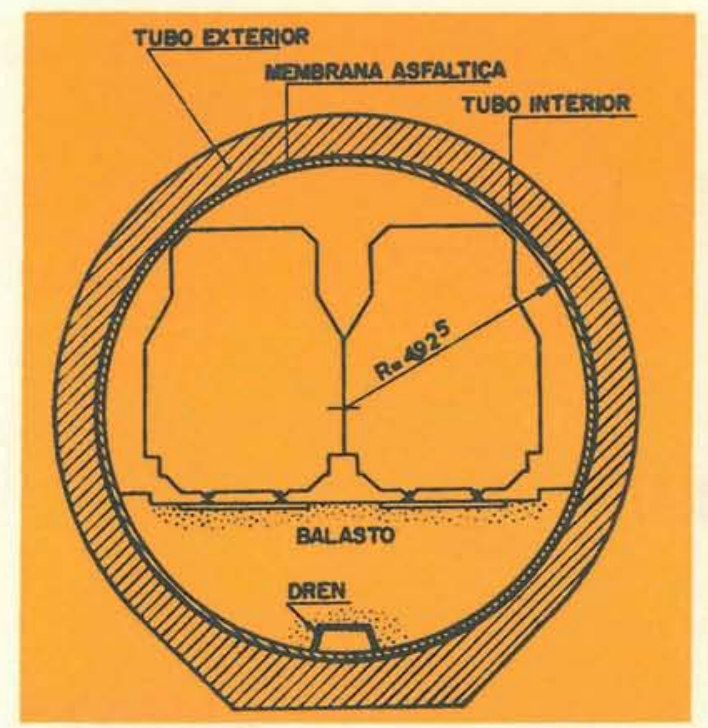

Fig. 9,-Túnel para ferrocarril.

Siguiendo este procedimiento, los distintos trozos del túnel se van uniendo entre sí en el dique seco o en la plataforma flotante para después ser transportados y hundidos en los lugares previstos siguiendo los métodos de hundimiento ordinarios o el llamado método de trinchera.

\section{Passadores especiules}

Como puede apreciarse en la figura 11, el dispositivo general de estos pasadores especiales está constituido por un cuerpo formando base, en forma de vaso, con alas, en forma de anillo, y que arrancan en su altura media, cubierto por una capa cuyas parades se ajustan con gran precisión a la parte externa del vaso, al que recubren, y provista también de alas que, a su vez, se ajustan sobre las partes exteriores de las alas anulares correspondientes al vaso. El conjunto de este dispositivo, denominado pasador especial, tiene por objeto solidarizar el tubo exterior con el interior, finalidad que se logra y ajusta por medio de un flete al que se le acopla una arandela y tuerca de presión.

Las superficies deslizantes entre vaso y tapadera han sido terminadas mecánicamente con gran precisión, y se lubrican con un producto llamado "molykote".

Estos pasadores especiales se suministran en obra completamente ajustados y montados, y debe tenerse la precaución de no separar en ningún momento el vaso de la tapa que le cubre. La unión entre estas dos partes es casi perfecta sin espacio libre.

La parte de asfalto que está comprendida entre las dos alas del pasador especial, se impermeabiliza por medio de la presión que la maniobra de la tuerca proporciona. Una capa suplementaria de asfalto sobre las referidas alas del pasador evita toda penetración posible de humedad. La acumulación del polvo que la oxidación o corrosión ocasiona entre las superficies en contacto podría dar lugar a la pérdida de la buscada impermeabilización, por lo que se ha colocado una capa suplementaria anular de espuma plástica, impregnada, entre tapa y vaso, durante el tiempo que dichos pasadores se hallan en la factoría.

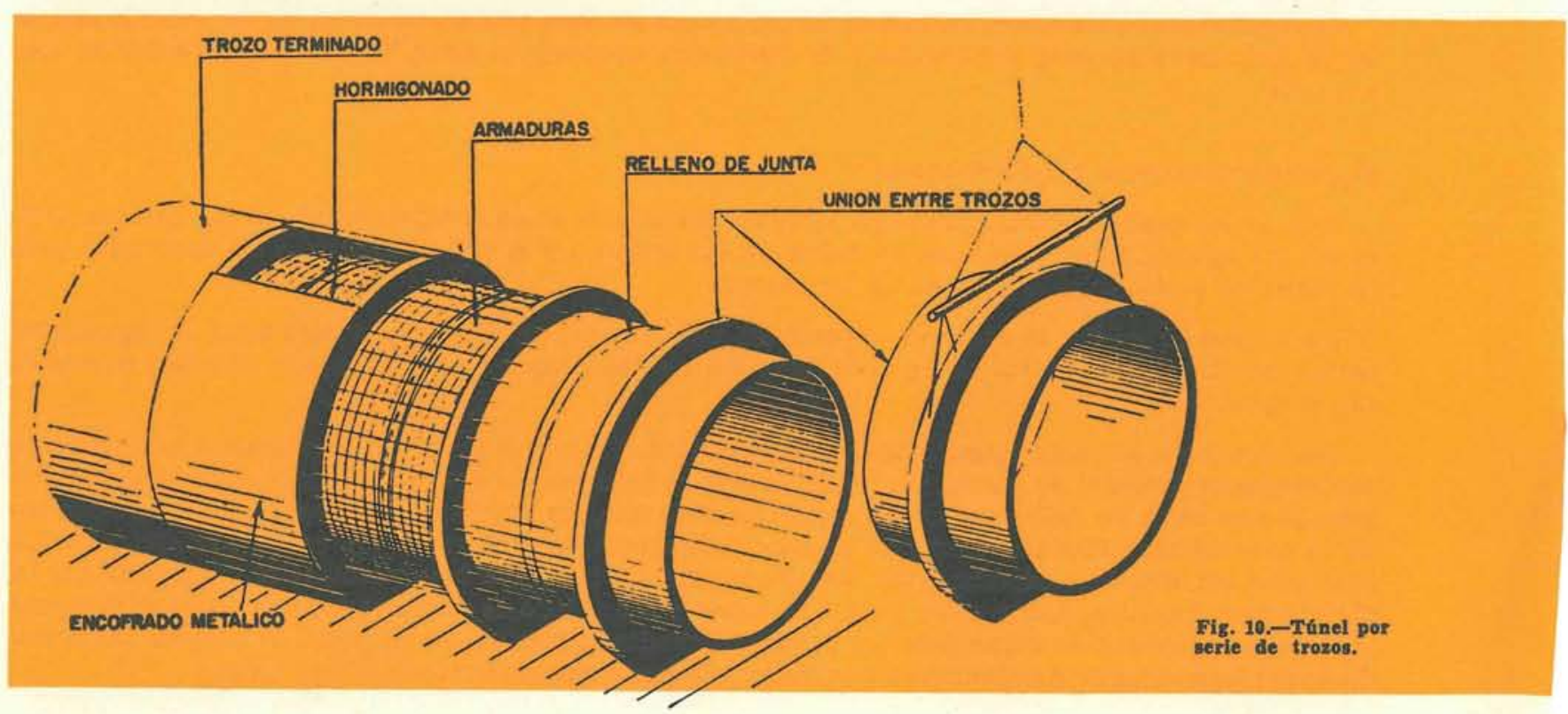




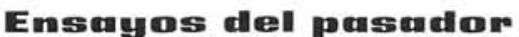

Se construyeron dos tipos distintos de estos pasadores y se sometieron a continuados ensayos dirigidos por el ingeniero W. J. Beranek, del Instituto de Materiales de Construcción y Construcción del Consejo Superior de Investigaciones Científicas de Holanda. Estos dos tipos de pasadores especiales, designados con la denaminación $A$ y $B$, fueron construidos con los materiales indicados en el cuadro I y con características tecnológicas que se indican en el cuadro II.

Estos pasadores especiales se sometieron a distintos ensayos: unos de resistencia, otros de rigi$\mathrm{dez}$, respecto a deformaciones tangenciales; rigidez respecto a la rotación; rozamiento entre las superficies del vaso y la tapa en movimientos de tipo radial con y sin rotación entre el tubo interior y exterior; excentricidad en la transferencia de cargas y, finalmente, fueron sometidos a cargas de rotura bajo diferentes circunstancias.

El resultado más importante de estos ensayos ha sido el que se refiere al rozamiento, que se eleva de un 20 a un 25 por 100 , y la carga de rotura, que ha resultado ser de 60 toneladas.

\section{Gompontramiento de los puscudomes}

El hecho de que el vaso puede moverse libremente dentro de la tapa tiene un doble efecto, ya que, en primer lugar, se logra casi eliminar los
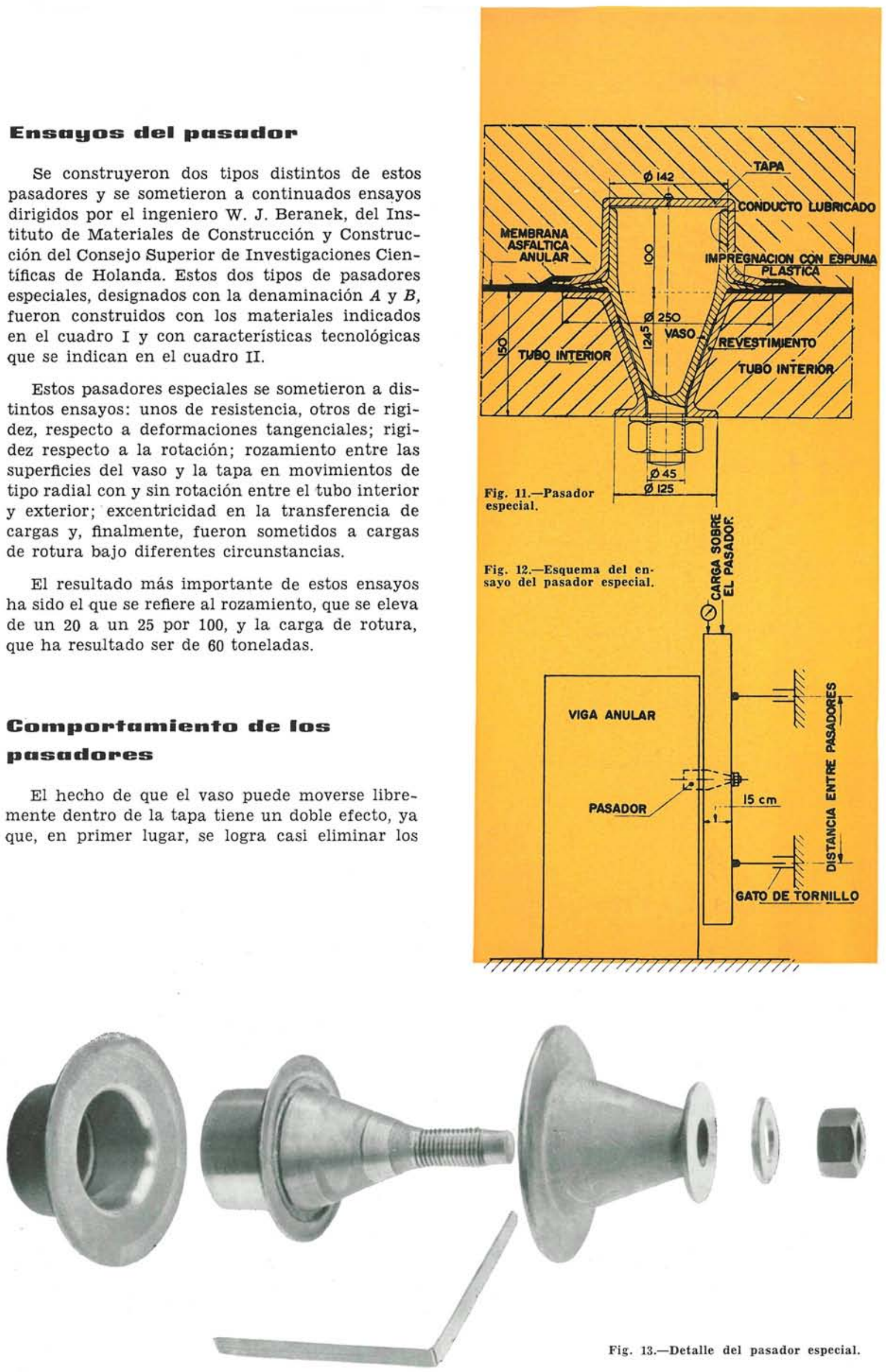

Fig. 13.-Detalle del pasador especial. 
CUADRO I

COMPOSICION DE LAS PARTES DEL PASADOR

\begin{tabular}{|c|c|c|c|c|}
\hline \multirow[t]{2}{*}{ Denominación } & \multirow[t]{2}{*}{ P A R T E } & \multicolumn{3}{|c|}{$M A T E A I A L$} \\
\hline & & $\begin{array}{c}\text { Ferrítico } \\
\text { Nodular } \\
\text { Fundición }\end{array}$ & $\begin{array}{c}\text { Perlitico } \\
\text { Nodular } \\
\text { Fundición }\end{array}$ & $\begin{array}{l}\text { Perlítico } \\
\text { Fundición }\end{array}$ \\
\hline A & 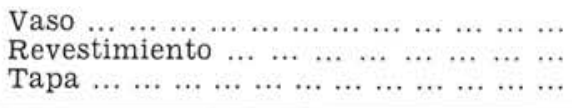 & & + & $\begin{array}{l}+ \\
+\end{array}$ \\
\hline B & & + & & $\begin{array}{l}+ \\
+\end{array}$ \\
\hline
\end{tabular}

CUADRO II

CARACTERISTICAS TECNOLOGICAS

\begin{tabular}{|c|c|c|c|}
\hline MA T ER I A L & $\begin{array}{l}\text { Punto de fluencia } \\
\left.\mathrm{kg} / \mathrm{cm}^{2} \text { (libras } / \text { pulg }^{2}\right)\end{array}$ & $\begin{array}{l}\text { Resistencia a tracción } \\
\left.\mathrm{kg} / \mathrm{cm}^{2} \text { (libras } / \mathrm{pulg}^{2}\right)\end{array}$ & $\underset{\%}{\operatorname{Alargamiento}}$ \\
\hline 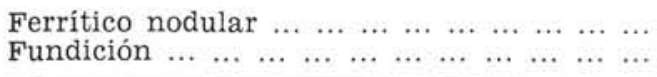 & $3.500(49.800)$ & $\begin{array}{c}4.200-4.800 \\
(59.700-68.300)\end{array}$ & 20 \\
\hline 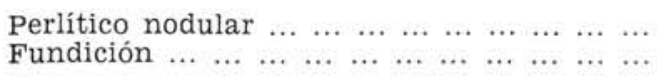 & $4.500(64.000)$ & $\begin{array}{c}5.500-6.000 \\
(78.200-85.300)\end{array}$ & 5 \\
\hline 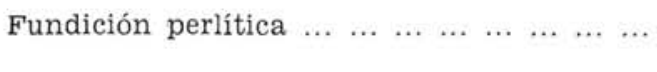 & - & $\begin{array}{c}2.200-2.600 \\
(31.300-37.000)\end{array}$ & - \\
\hline
\end{tabular}

efectos perturbadores de la flexión en la dirección longitudinal del túnel $\mathrm{y}$, en segundo lugar, que la unión cerrada entre el tubo exterior e interior, al ser íntima, daría lugar a distorsiones que dependerían de la profundidad del agua sobre el túnel y que pronto podrian elevarse a valores inadmisibles. Puesto que los pasadores permiten un movimiento radial entre los dos tubos, las distorsiones antes mencionadas dependen, únicamente, de los rozamientos de dirección radial cuya cuantía es del 20 al 25 por 100 del esfuerzo tangencial. Como el esfuerzo de estos pasadores no depende de la profundidad del agua sobre $\mathrm{el}$ túnel, la distorsión en los bordes tampoco depende de la misma. Los esfuerzos de flexión debidos a la distorsión en el borde son directamente proporcionales a las tensiones radiales; $y$ el valor de estas tensiones es de $100 \mathrm{~kg} / \mathrm{cm}^{2}$ y de $40 \mathrm{~kg} / \mathrm{cm}^{2}$ las tangenciales.

Fig. 14.-Banco de ensayos del pasador

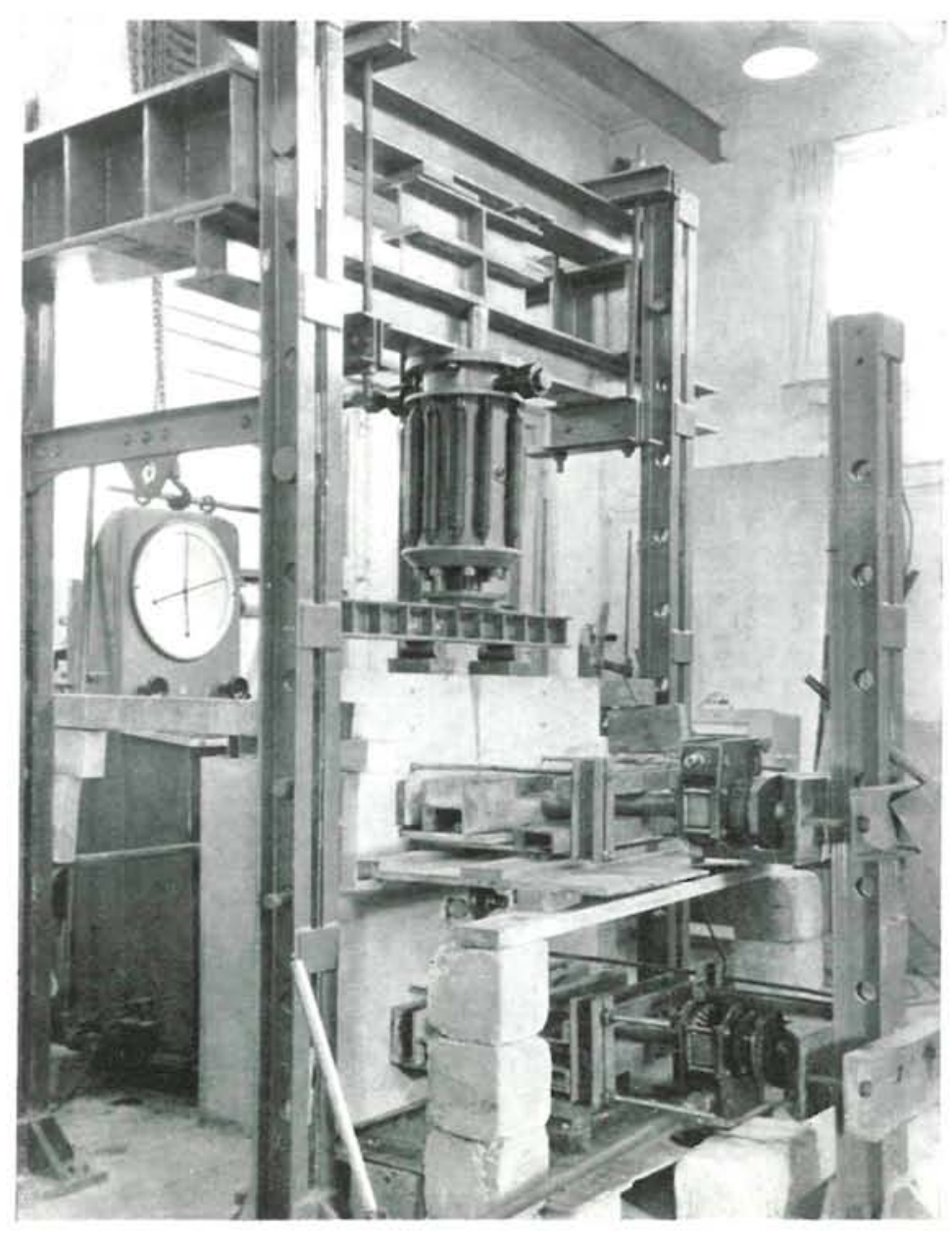



han colocado los pasadores tiene $18 \mathrm{~cm}$ de refuerzo con el fin de limitar las tensiones motivadas por flexión a $20 \mathrm{~kg} / \mathrm{cm}^{2}$.

Durante la fase constructiva la viga en forma de anillo se halla sometida a momentos flectores. En el caso en que las paredes del tubo formasen un conjunto monolítico con el anillo actuarian como alas sobre una cierta zona y, por lo tanto, experimentarían las tensiones correspondientes a las fibras externas. El tubo interior, no obstante, tiene una libertad radial de movimiento respecto al anillo o viga anular del tubo exterior. El tubo interior evita, por consiguiente, la acción conjunta con el tubo exterior y, de tal forma, que el alargamiento circunferencial del tubo es casi nulo y de acuerdo con la ley de trabajo mínimo. El tubo constituye, por sí solo, una lámina rígida respecto a las deformaciones en su propio plan.

Además de las deformaciones debidas a las flexiones en dirección axial del túnel, se logra reducir la distorsión de dirección circunferencial como consecuencia de la libertad de movimiento en dirección radial de los pasadores. La transmisión de los esfuerzos tangenciales, de principal importancia para el conjunto interior, se ha realizado por medio de los pasadores.

\section{Béllculo dell tulbo imtemiom}

El profesor A. L. Bouma y H. van Koten, del Instituto anteriormente mencionado, fueron encargados de calcular las tensiones y deformaciones de los distintos trozos del tubo interior durante las diferentes fases de construcción y para después entrar en servicio la obra.
El espesor de la pared del tubo en el lugar donde se
Fig. 15.-Diagrama de cargas del pasador.

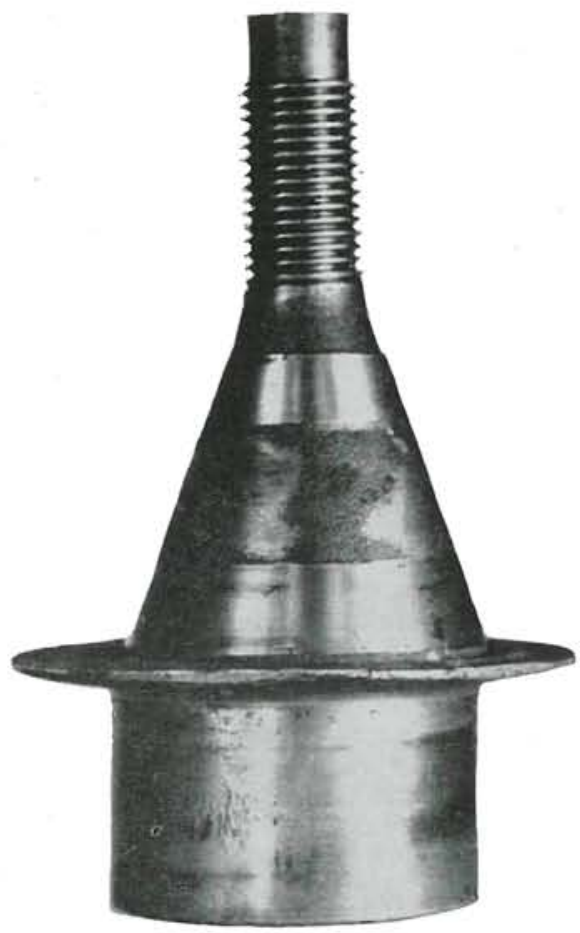

Fig. 17.-Vaso del pasador.

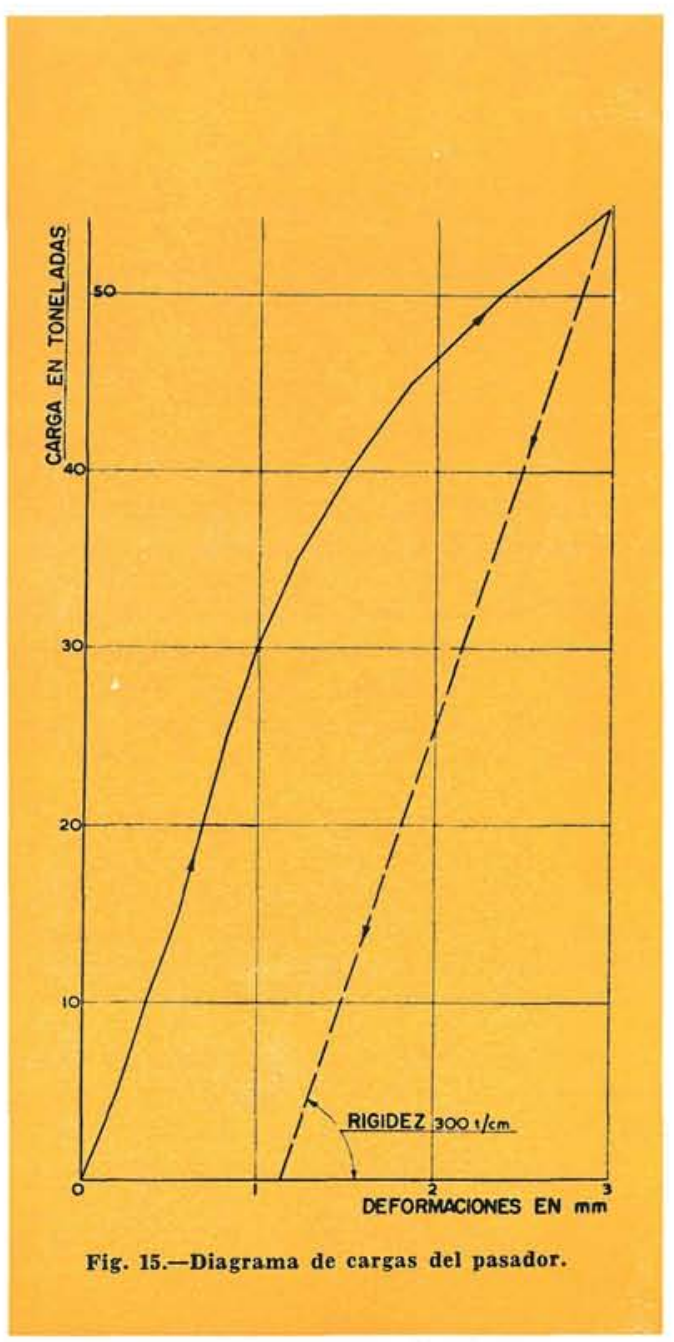

Fig. 16.-Tapa del pasador.
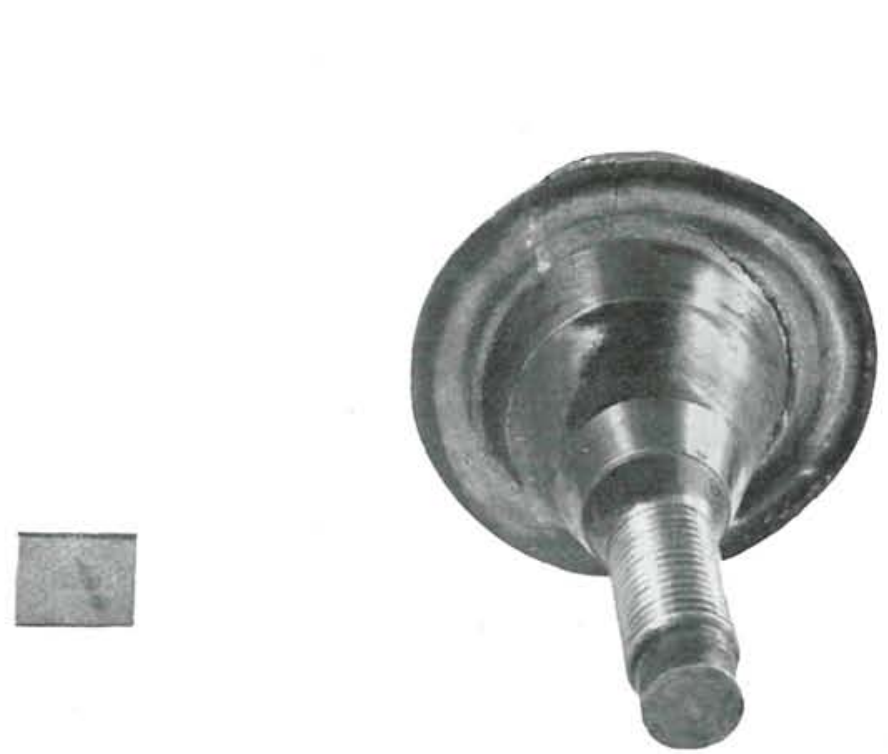


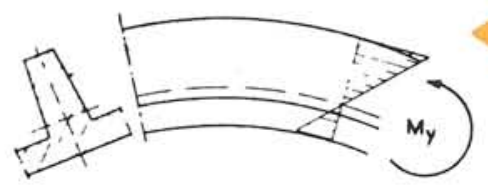

Fig. 18.-Esfuerzos en tubos unidos con pa. sadores.

Fig. 19.-Esfuerzos tangenciales al pasador son favorables para flexiones.

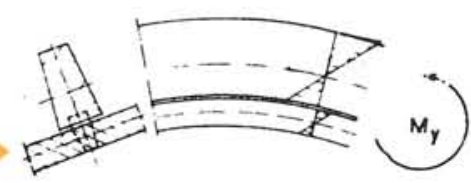

Las bases que han servido para estos cálculos se han sentado en los principios publicados en distintos informes, mientras que las características de los pasadores especiales, obtenidas como consecuencia de los ensayos antes referidos, han sido tenidas en cuenta en lo concerniente a la rigidez respecto a esfuerzos cortantes y rotaciones. Con relación a las fuerzas de rozamiento de dirección radial en los pasadores se han hecho cálculos adicionales, puesto que al carecer de linealidad y reversibilidad estas fuerzas de rozamiento no se pueden utilizar como condiciones en el borde de los cálculos de las láminas antes referidas. Los resultados más importantes de la investigación en este sentido se pueden resumir así:

\section{a) En el caso de una sección en posición horizontal.}

En este caso la viga anular se halla sometida a tensiones locales de flexión de un valor máximo de $67 \mathrm{~kg} / \mathrm{cm}^{2}$, por lo que aparece la fisuración en la zona de tracciones. Por todo ello, la rigidez del anillo disminuye de tal forma que las deformaciones aumentan y las tensiones de flexión en el tubo pueden alcanzar hasta $10 \mathrm{~s} 40 \mathrm{~kg} / \mathrm{cm}^{2}$.

Por sí mismo, estas tensiones no son significativas en lo que respecta a resistencia. Se han admitido tensiones de flexión de $80 \mathrm{~kg} / \mathrm{cm}^{2}$ tratándose del hormigón armado, pero, con una tensión de $40 \mathrm{~kg} / \mathrm{cm}^{2}$ la fisuración en la zona sometida a tracción es inevitable. Cuando es necesario obtener un tubo interior impermeabilizado por sí mismo y en el que la capa asfáltica de impermeabilización puede considerarse como una seguridad complementaria, la viga anular debe asegurarse contra la fisuración dándole mayores dimensiones o también por medio de pretensado o proporcionando a los puntos de apoyo mayor separación.

Las tensiones finales de flexión son muy pequeñas. El estado tensional presenta pequeñas diferencias respecto a los estados tensionales en las membranas, en cuyo caso $M_{y}=0$. Además, todas las tensiones debidas a la flexión se hallan ampliamente atenuadas por la presión circundante del agua.

\section{b) Tensiones normales de dirección circunferencial.}

Como se ha indicado en la adjunta figura (fig. 8), difieren muy poco del estado tensional en una membrana, motivado por el peso propio y la presión del agua.

\section{c) Esfuerzo cortante absorbido por los pasadores.}

Su valor máximo es casi igual al obtenido en un estado tensional de membrana debido al peso propio y a la presión del agua; sin embargo, debido a las cargas de las partes interiores de la construcción y al tráfico, aparece cierta traslación en la posición del máximo.

El coeficiente de rotura es, por lo menos, 3. La zona sombreada de la figura 8 adjunta indica la influencia máxima de las cargas debido al tráfico en los esfuerzos de los pasadores.

\section{d) Estabilidad.}

Se ha hallado una tensión de $90 \mathrm{~kg} / \mathrm{cm}^{2}$ teniendo en cuenta el pandeo en los cálculos de estabilidad elástica. Esto indica que el peligro de pandeo solamente se habría agudizado con una profundidad de agua de $900 \mathrm{~m}$, en cuyo caso la resistencia a la rotura del hormigón se halla largamente sobrepasada.

Naturalmente, el fenómeno plástico puede aparecer antes; sin embargo, la seguridad contra los efectos del pandeo resulta, en general, más que suficiente.

Los cálculos de estabilidad, de acuerdo con la teoría de Flügge, son aplicables a vigas cilíndricas de tipo tubular rígidas. Los trozos de túnel se unen con las vigas rígidas por medio de pasadores especiales que permiten un movimiento radial. En el caso de pandeo no simplemente en el sentido radial, sino en el tangencial, los corrimientos se ponen de manifiesto. Estos últimos pueden evitarse utilizando los pasadores especiales. El estado general se presenta, pues, como si se tratase de vigas rígidas. 


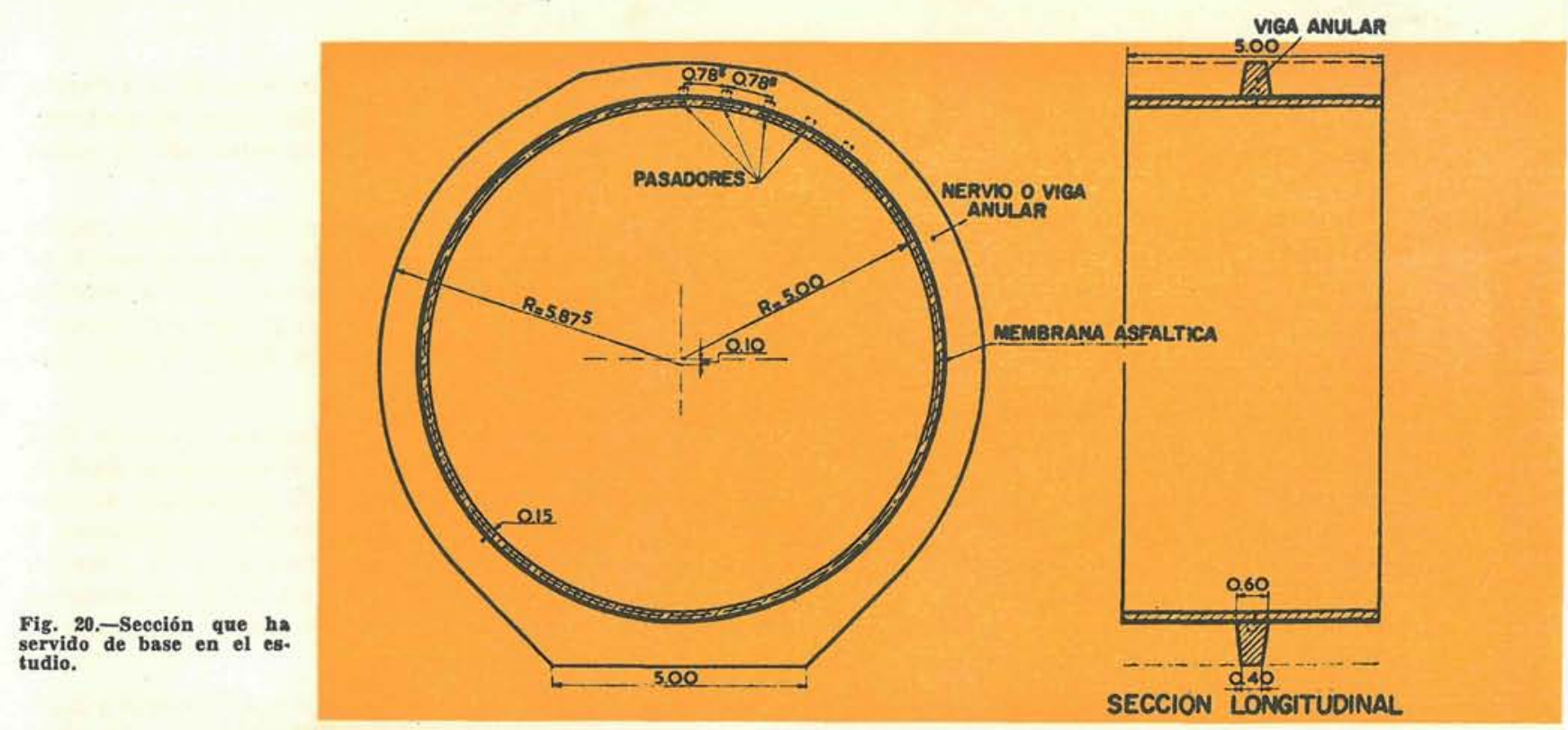

\section{Juntatas}

Tratándose de un método constructivo que se apoya en la prefabricación de elementos, las juntas requieren especial cuidado. Cuando se unen los elementos entre sí por medio de barras pretensadas o cables, se procura, generalmente, rellenar las juntas con un material de la misma resistencia que los propios elementos.

Por ello, en el caso que nos ocupa, se hace innecesario en el proyecto tal procedimiento y hasta indeseable. Cuando se coloca un túnel sobre terreno blando o aparecen capas compresibles, por debajo aparece una expansión en el fondo después de haber terminado el dragado de la trinchera dentro de la cual ha de colocarse el túnel. Por todo ello, los trozos del túnel se transportan al lugar de su ubicación, y acto seguido de haberlos colocado se rellena la trinchera. El empuje del fondo en estas condiciones se atenúa parcialmente, puesto que el túnel pesa menos que la parte original de tierras excavadas. Esto implica que la consolidación se realiza en la zona sobre la línea $A$ y no en la zona correspondiente a la línea $C$, de pendiente más pronunciada, si es que se acepta la ley de consolidación de Terzaghi, como indicación de lo que se puede esperar. Además, en muchos casos no se alcanza el empuje total durante el pequeño período de tiempo en que se ha abierto la trinchera para la colocación del tubo. Naturalmente, el fenómeno resulta ser mucho más complicado y siempre requiere una investigación especial que afecta también a los cimientos del pozo de la estación de ventilación. De todo este análisis resulta evidente que los asientos diferenciales permanecen casi siempre pequeños, sin que sea necesario de otros requerimientos para las normas de ejecución que el de rellenarlas en las inmediaciones del túnel uniformemente en todas las partes.

La experiencia obtenida en numerosos túneles conflrma esta aseveración. Existen, naturalmente, excepciones, pues puede ocurrir que los asientos diferenciales esperados sean tan grandes que tengan que utilizarse métodos diferentes para mantener los cimientos dentro de ciertos límites como, por ejemplo, el empleo de pilotes para cimientos.

Los costes que así se hallan envueltos bajo estas condiciones plantean la cuestión de determinación de la necesidad de tales procedimientos. La contestación a estas cuestiones depende, no solamente de la extensión esperada del asiento diferencial, sino de la relación en que con ello se halla la rigidez del túnel en su dirección longitudinal. Indiferentemente de los caminos para determinar estos factores, ya por medio de cálculos o con ayuda de criterios obtenidos en ensayos sobre modelos reducidos, hay que tener siempre en cuenta que las deformaciones se conserven dentro de tales límites que:

a) Las tensiones permitidas en la estructura no deben ser excedidas.

b) La impermeabilización no debe hallarse en peligro.

c) El perfll longitudinal de la calzada o plataforma para vía férrea en el túnel debe permanecer en buenas condiciones. 
En la primera condición aparecen tensiones como consecuencia de la ventilación durante la estación fría que rebajan la temperatura en el interior del túnel, mientras que la exterior del suelo es superior, con lo que se presenta un estado favorable para la fisuración, por lo que el túnel se subdivide en trozos y han de revisarse las cargas y tensiones.

En el caso en que la impermeabilización y la uniformidad de la calzada o vía férrea no se hallen en peligro, este estado de cosas no resulta alarmante, pero, si las deformaciones esperadas son tales que el proyecto de ejecución no presenta la conflanza debida en vista de los criterios a que se refleren los apartados anteriores b) y c), entonces la solución la daría una cimentación con pilotes. Así se puede lograr la rigidez necesaria en el túnel, si bien aumenta al mismo tiempo la carga debida a las capas de relleno superpuestas al túnel.

Esta última condición, sin embargo, constituye un fenómeno común en la mecánica del suelo. Una construcción rígida maniflesta cierta atracción de la carga, mientras que la repele si aquélla es elástica. En el túnel subdividido en partes se intenta sacar partido de este fenómeno dando flexibilidad al tubo interior. Los elementos se aprietan entre sí por medio de algunos pasadores rectilíneos sometidos a grandes tensiones e insertando anillos de goma como empaquetadura. De esta forma la parte superior flexible, de impermeabilización del túnel, se pone en acción y permite los movimientos de deformación motivados por el asiento y diferencias de temperatura a través de los referidos anillos o juntas de goma que constituyen discos cartilaginosos.

La resistencia de los trozos de túnel y en sentido longitudinal, necesaria durante el transporte y hundimiento de dichos trozos, se logra por medio de armaduras longitudinales colocadas en el tubo exterior. La condición final esencial, para la resistencia, es que los esfuerzos cortantes puedan ser transferidos entre la viga anular y el tubo exterior. Por lo tanto, la superficie lateral de dichos anillos va provista de arandela. De esta forma, al oxidarse la armadura longitudinal en el curso de los años es posible mantener por este procedimiento la resistencia.

Para la determinación final de dimensiones y rigidez de los anillos de guarnición, sólo ha de considerarse el criterio mencionado en el apartado c) que se refiere a la cuestión de la necesidad de una cimentación con pilotes.

Finalmente, se señala que, en las zonas expuestas a sismos, un túnel con tubo flexible tiene mayor importancia, puesto que las posibilidades de fisuración y anegación son menos probables que en caso de un tubo rígido.

\section{Observeruciomes fincalles}

Después de mayor maduración, el proyecto original, más o menos intuitivo, resultó ser, en ciertos aspectos, una continuación de los nuevos desarrollos referentes a la construcción de túneles, especialmente en los Estados Unidos. Estos desarrollos implican los métodos de prefabricación, así como el cálculo, de acuerdo con la teoría de los grandes oleoductos. En los últimos proyectos americanos, esta teoría se ha aplicado a los cálculos de tubos de hormigón para determinar los programas de hormigonado del revestimiento interior.

El túnel constituido por trozos sucesivos se considera como si se tratase de un oleoducto en todas sus fases constructivas y, además, como una estructura; por tanto, para los efectos de cálculo, se hace uso de los nuevos conceptos, de acuerdo con los trabajos consultados y de reciente publicación. De estos conceptos resulta evidente que las tensiones máximas debidas a la flexión en el tubo interior no pasan de los 20 a $25 \mathrm{~kg} / \mathrm{cm}^{2}$, mientras que la solución de simple membrana, considerando la carga por peso propio y presión del agua, da una buena aproximación del estado final tensional.

Un nuevo desarrollo de ideas es el de la función atribuida a la separación entre tubos, por lo que éstos se proyectan para su peso propio y presión del suelo, por una parte, y para la presión del agua, por otra. Los ensayos ponen de manifiesto que el elemento proyectado para la conexión e interacción entre los dos tubos, es decir, el pasador especial radial, se comporta de acuerdo a lo previsto y con un gran margen de seguridad.

La aplicación de los métodos de prefabricación en la ingeniería hidráulica permanece aún dentro de un campo no explotado. Es de esperar que un desarrollo ulterior conducirá a nuevos conceptos, para los que no bastan los intentos tradicionales y los que cada día se orientan más hacia la preparación del proyecto, detalles y métodos constructivos basados en la prefabricación.

Las posibles aplicaciones del sistema de túnel por trozos sucesivos se van acentuando cada día con mayor intensidad. Respecto a este particular y posibles reducciones del coste, se confía en que pronto aparezca más información. En varios países se han patentado ya nuevos procedimientos constructivos. 


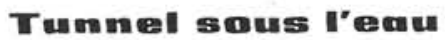

E. Stambuis, ingénieur.

Ce travail analyse les résultats obtenus d'une étude théorique effectuée par l'Institut Central de Recherches Scientifiques de Hollande sur les tunnels submergés, afi
avantages sur ceux actuellement existant.

En primer lieu, sont étudiées la résistance, l'étanchéité et la ventilation les plus rationnelles pour la rédaction du projet d'exécution.

Ensuite, sont analysées la forme, les sections appropriées et les méthodes constructives en les comparant avec celles utili. sées dans la construction d'autres tunnels de cette nature en Amérique. Une attention partículière est attachée aux procédés pour lesquels la ligne générale constructive est basée sur la préfabrication et le montage des éléments structuraux préparés en atelier, leur transport et leur mise en oeuvre.

Comme détails complémentaires, l'auteur s'étend en considérations sur les jeux des pressions du sol et de l'eau, poussées en flotaison et les cas où apparaissent des flexions et des efforts tranchants dans les différents tronçons qui divisent la en flotaison et les cas où appar
longueur totale du tunnel.

L'auteur s'efforce de donner un caractère pratique aux applications qui peuvent découler de 1'étude théorique, pour la réalisation de l'ouvrage.

Sont décrits également les goujons spéciaux qui servent à assembler les deux tubes qui composent le tunnel, les joints entre deux troncons différents, leur étanchéité et la flexibilité qu'ils permettent. Finalement, l'auteur résume son exposé théorique avec une série d'observations et de conseils applicables à ces constructions.

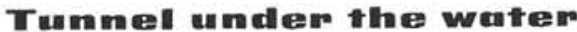

E. Stambuis, engineer.

This article describes the results obtained by the Dutch Central Institute of Scientific Research when studying the problem of underwater tunnels. This Institute has sought to establish general specifications for the design of this type of tun nels, so that they shail be more efficient than those at present in use.

Firstly, a study was made of the strength, waterproof qualities, and most rational type of ventilation, with a view to drawing up of general principles on this aspect of the design.

An analysis is given of the shape, suitable cross sections and constructive methods, and these are compared with those adopted in the construction of underwater funnels at present in use in America. Particular attention has been given to and erection of these at the site. The author goes on to discuss the interplay of soil and waterpressures, buoyancy forces, and cases in which the tunnel is
subjected to important bending and shear forces on particular sections of it. In this connection care is taken to give the theoretical considerations a ready practical interpretation.

In particular, a description is given of special distance pieces which connect the two tubes making up a tunnel, also the system of joining two sections of the tube, and the measure of watertightness and flexibility which such joints provide. Finally, a summary is given of the theoretical foundations, as well as a number of observations and items of advise, which are applicable to these types of structures.

\section{Unt}

E. Stambuis, Ingeniers.

In dieser Arbeit werden die Resultate einer theoretischen Studie des Zentralinstituts für wissenschaftliche Forschung in In eine vorteilhaftere Planung dieser Art von Tunnel erlauben.

Zunächst werden die Widerstandsfähigkeit und die rationellere Abdichtung und Lüftung des geplanten Projektes untersucht.

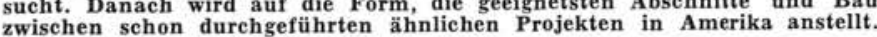

Besondere Aufmerksamkeit wird der Fertigbauweise, dem Transport der vorfabrizierten Teile und deren Montage aut der Baustelle gewidmet.

Zusätzlich stellt der Verfasser Uberlegungen über das Zusammenspiel zwischen Boden- und Wasserdruck und den Auftriebskräften an und betrachtet die Fälle, in denen Biege- und Schubspannungen in den verschiedenen Abschnitten des Tunnels auftreten.

Ausserdem bemüht sich der Verfasser, den theoretischen Ausführungen eine praktische Anwendung für die Durchführung des Projektes su geben.

Ferner werden einige Spezialdübel beschrieben, die zur Befestigung der beiden Tunnelrohre dienen; desgleichen behandelt der Verfasser die Abdichtung der Fugen und die damit erreichte Biegsamkeit. Zum Schluss bringt der Author noch eine Zusammenfassung seiner theoretischen Ausführungen mittels einer Reihe von Empfehlungen und Ratschläge für den Bau soleher Projekte. 